\title{
Pediatric lung ultrasound cons - are they really strong enough?
}

\author{
Jovan Lovrenski ${ }^{1}$
}

Received: 12 September 2019 /Revised: 12 September 2019 / Accepted: 26 September 2019/Published online: 13 February 2020

(C) Springer-Verlag GmbH Germany, part of Springer Nature 2019

In his article "Lung ultrasound in pediatric radiology - cons" [1], Paolo Tomà has excellently gone through the publishing history of articles dealing with thoracic ultrasound (US) from 1975 to the present. An increasing number of publications, especially from the mid-1990s to now, demonstrate the increasing interest in this diagnostic tool. There is an old proverb in my country saying "a huge cloud of dust always follows a good horse." When there is so much recent talk about lung US, there has to be something worthy about it. However, there is something intriguing and incomplete about it, too, otherwise its impact on everyday clinical practice would not be persistently questioned. I have a feeling that we are circling around the same story over and over again. It is obvious that lung US is not a perfect, self-sufficient diagnostic tool. It is not possible to assess central airways, hilar and subscapular regions and to detect any pathological entity that does not abut the pleura. Lines, tubes and some air leak phenomena are also problematic, as Tomà emphasizes. Yet lung US exists with all its imperfections, and numerous studies report its strong impact on everyday clinical work, with high sensitivity and specificity $[2,3]$, which cannot be explained as a coincidence. The applications of lung US most frequently studied are pediatric pneumonia and neonatal lung disease and their complications.

There is one extremely valuable characteristic of lung US that I realized throughout my use of it on a daily basis: Lung US does not lie. When something is seen on lung US, it really exists. The only thing that can lie is the interpretation of findings, and in this respect I very much agree with Tomà. We should not be afraid to state in our reports that lung US findings are nonspecific. And it is the greatest wisdom of all not to pretend that lung US is something that it is not - an almighty

Jovan Lovrenski

JOVAN.LOVRENSKI@mf.uns.ac.rs

1 Faculty of Medicine, Radiology Department, Institute for Children and Adolescents Health Care of Vojvodina, University of Novi Sad, Hajduk Veljkova 10, Novi Sad 21000, Serbia diagnostic tool. When we observe a small area of interstitial edema or subcentimeter (particularly single) subpleural consolidation, especially if there is no specific clinical context, we simply should not be so arrogant as to claim it is early pneumonia, small atelectasis or something else, or try to differentiate the nature of infection. So, lung US can be compromised only by lung US operators, whether clinicians or radiologists. Maybe Tomà is right that clinicians "fly high" when they sense all the benefits that lung US offers to them, particularly in emergency departments and intensive care units. However, I truly think our clinical colleagues have good intentions. They may just need to be pointed in the right direction. Radiologists should help them, not by showing their superiority (which is obvious and expected in respect to diagnostic imaging), but to point out the limitations of lung US and offer other diagnostic modalities that are more effective in certain situations. I do not believe that clinicians should follow "radiological culture"; we should keep the US signs as simple as possible, but within a reasonable framework of interpretation limits. Naturally, proper training is crucial, and it has to be defined more precisely in the future [4]. However, a difference in reliability between experts and inexperienced US operators is something that is expected, highly reasonable and logical not only in case of lung US, but for any other diagnostic modality. We have to be aware that high-quality training needs to be provided for lung US beginners. There is no such thing as an experience transplant. This is something that each operator has to go through on their own to reach a certain level of expertise (for example, to be able to confidently differentiate between fluid within abscess and focal pleural effusion, you need to see it several times and go through solving this dilemma yourself).

My experience shows that US diagnosis of pneumothorax is the most difficult task in neonates and very young children in general. Their breathing pattern is unique; they often have apneic periods, periods of shallow breathing, etc. This sometimes makes detecting lung sliding exceptionally hard. Experience is critical. Inexperienced operators need to take one step at the time. Their first achievement should be to use 
lung US for follow-ups, especially after thoracocentesis. If they see B-lines, subpleural consolidation(s), and/or lung sliding, this should be interpreted as definite signs of lung reexpansion, i.e. signs that exclude pneumothorax. Even this first step would certainly reduce the number of chest radiographs, especially in neonatal intensive care units. We also have to be honest and admit that the estimation of the quantity of pneumothorax is quite limited on US.

Misinterpretation and lack of experience can definitely lead to antibiotics overuse. Therefore, we need to understand that residual lung pathology can be seen on US for days, weeks, sometimes even months before complete regression, as is the case on chest radiographs. The normalization of laboratory and clinical findings suggests that small pathological areas detected by lung US are probably not clinically important.

The duration of a lung US exam is also very closely related to the operator's experience. It most often takes no more than 5 to $6 \mathrm{~min}$, but in inexperienced hands it can be as long as $15 \mathrm{~min}$. However, this changes as soon as the lung US operator gains confidence. When we talk about the cost-benefit ratio, it seems that lung US performed by radiologists in ambulatory settings makes more sense both from the aspect of fast decision-making (proper triage of patients with pneumonia or pneumothorax, for example) and from a financial aspect. On the other hand, bedside lung US examinations at various pediatric/pediatric surgery departments or intensive care units are highly questionable from a financial perspective. Those examinations are very time-consuming for radiologists due to the combination of (a) complex patients who need to be examined longer and (b) the time that has to be spent going back and forth between the different departments, talking to clinicians most often both before and after the lung US exam, and writing the lung US report away from the radiology department. This is probably the reason clinicians are increasingly taking over this examination from radiologists. There is also one more important thing: Clinicians are best acquainted with patients who need bedside examinations and can easily correlate clinical and imaging findings. However, this is a mixed blessing. Knowing all the clinical facts, clinicians might falsely interpret lung US findings in the way that best fits the patient's clinical condition. This can be avoided if clinicians are sufficiently alert to bias.

An observation in Tomà's article that the use of contrast agent does not add much to Doppler evaluation is something I agree with, except in the very early stages of necrotizing pneumonias where areas of necrosis occasionally are not clearly demarcated on lung US. In such cases, the application of contrast medium can help to differentiate area(s) of necrosis easier and clearer than by using both gray-scale and Doppler US. It is a different question whether we should perform a contrast- enhanced US in early complicated pneumonias considering its cost, especially in under-resourced countries. Intracavitary contrast-enhanced US can be useful as well. It can determine the position of a chest catheter, its patency and the presence of loculations [5].

In conclusion, lung US is a diagnostic technique that can be compromised not by its imperfections, but by insufficiently trained US operators and their interpretation of findings. However, this is an issue with all new diagnostic tools. Lung US is one of the most easily learned diagnostic techniques today, with no more than 10 basic signs that can do the job and be useful in pediatric clinical practice. Why do we so often have a tendency to make simple things complicated? Instead of making it a flaw of the method, we should make it its greatest advantage, as it really is. Our goal needs to be to create adequate learning and teaching guidelines, with a worldwide consensus on standardization of technique and interpretation. I strongly believe that the wide availability, the mobility of US, the fact that modern US devices are not required, the relative simplicity of the lung US itself, its high reliability proven in a number of studies and its radiation-free nature would at least maintain a balance against all the cons on a pair of scales. Obviously, there are very reasonable counterarguments, but not nearly enough to make us give up on lung US now when it is finally receiving some well-deserved attention.

\section{Compliance with ethical standards}

Conflicts of interest None

\section{References}

1. Tomá P (2019) Lung ultrasound in pediatric radiology-cons. Pediatr Radiol. https://doi.org/10.1007/s00247-019-04524-z

2. Pereda MA, Chavez MA, Hooper-Miele CC et al (2015) Lung ultrasound for the diagnosis of pneumonia in children: a meta-analysis. Pediatrics 135:714-722

3. Balk DS, Lee C, Schafer J et al (2018) Lung ultrasound compared to chest X-ray for diagnosis of pediatric pneumonia: a meta-analysis. Pediatr Pulmonol 53:1130-1139

4. Pietersen PI, Madsen KR, Graumann O et al (2018) Lung ultrasound training: a systematic review of published literature in clinical lung ultrasound training. Crit Ultrasound J 10:23

5. Deganello A, Rafailidis V, Sellars ME et al (2017) Intravenous and intracavitary use of contrast-enhanced ultrasound in the evaluation and management of complicated pediatric pneumonia. J Ultrasound Med 36:1943-1954

Publisher's note Springer Nature remains neutral with regard to jurisdictional claims in published maps and institutional affiliations. 